\title{
On Peak versus Average Interference Power Constraints for Protecting Primary Users in Cognitive Radio Networks
}

\author{
Rui Zhang
}

\begin{abstract}
This paper considers spectrum sharing for wireless communication between a cognitive radio (CR) link and a primary radio (PR) link. It is assumed that the CR protects the PR transmission by applying the so-called interference-temperature constraint, whereby the CR is allowed to transmit regardless of the PR's on/off status provided that the resultant interference power level at the PR receiver is kept below some predefined threshold. For the fading PR and CR channels, the interference-power constraint at the PR receiver is usually one of the following two types: One is to regulate the average interference power (AIP) over all the fading states, while the other is to limit the peak interference power (PIP) at each fading state. From the CR's perspective, given the same average and peak power threshold, the AIP constraint is more favorable than the PIP counterpart because of its more flexibility for dynamically allocating transmit powers over the fading states. On the contrary, from the perspective of protecting the PR, the more restrictive PIP constraint appears at a first glance to be a better option than the AIP. Some surprisingly, this paper shows that in terms of various forms of capacity limits achievable for the PR fading channel, e.g., the ergodic and outage capacities, the AIP constraint is also superior over the PIP. This result is based upon an interesting interference diversity phenomenon, i.e., randomized interference powers over the fading states in the AIP case are more advantageous over deterministic ones in the PIP case for minimizing the resultant PR capacity losses. Therefore, the AIP constraint results in larger fading channel capacities than the PIP for both the CR and PR transmissions.
\end{abstract}

\section{Index Terms}

Cognitive radio, spectrum sharing, interference temperature, interference diversity, fading channel capacity.

\section{INTRODUCTION}

This paper is concerned with a typical spectrum sharing scenario for wireless communication, where a secondary radio, also commonly known as the cognitive radio (CR), communicates over the same bandwidth that has been allocated to an existing primary radio (PR). For such a scenario, the CR usually

Submitted to IEEE Transactions on Wireless Communications, June 1 2008, revised September 12008.

Rui Zhang is with the Institute for Infocomm Research, A*STAR, Singapore. (e-mail: rzhang@i2r.a-star.edu.sg) 
needs to deal with a fundamental tradeoff between maximizing its own transmission throughput and minimizing the amount of interference caused to the PR transmission. There are in general three types of methods known in the literature for the CR to deal with such a tradeoff. One is the so-called opportunistic spectrum access (OSA), originally outlined in [1] and later formally introduced by DARPA, whereby the CR decides to transmit over the PR spectrum only when the PR transmission is detected to be off; while the other two methods allow the CR to transmit over the spectrum simultaneously with the PR. One of them is based on the "cognitive relay" idea [2], [3]. For this method, the CR transmitter is assumed to know perfectly all the channels from CR/PR transmitter to PR and CR receivers and, furthermore, the PR's message prior to the PR transmission. Thereby, the CR transmitter is able to send messages to its own receiver and, at the same time, compensate for the resultant interference to the PR receiver by operating as an assisting relay to the PR transmission. In contrast, the other method only requires that the power gain of the channel from $\mathrm{CR}$ transmitter to $\mathrm{PR}$ receiver is known to the $\mathrm{CR}$ transmitter and, thereby, the CR is allowed to transmit regardless of the PR's on/off status provided that the resultant interference power level at the PR receiver is kept below some predefined threshold, also known as the interference-temperature constraint [4], [5]. In this paper, we focus our study on this method due to its many advantages from an implementation viewpoint.

To enable wireless spectrum sharing under the interference-temperature constraint, dynamic resource allocation (DRA) for the CR becomes crucial, whereby the transmit power level, bit-rate, bandwidth, and antenna beam of the CR are dynamically changed based upon the channel state information (CSI) available at the CR transmitter. For the single-antenna fading PR and CR channels, transmit power control for the CR has been studied in [6], [7] under the average/peak interference-power constraint at the PR receiver based upon the CSI on the channels from the CR transmitter to the CR and PR receivers, in [8] under the combined interference-power constraint and the CR's own transmit-power constraint, and in [9], [10] based upon the additional CSI on the PR fading channel. On the other hand, for the multi-antenna PR and CR channels, in [11] the authors proposed both optimal and suboptimal spatial adaptation schemes for the CR transmitter. Information-theoretic limits for multiuser multiantenna/fading CR channels have also been studied in, e.g., [12], [13]. 
In this paper, we consider the single-antenna fading PR and CR channels. For such scenarios, the interference-power constraint at the PR receiver is usually one of the following two types: One is the long-term constraint that regulates the average interference power (AIP) over all the fading states, while the other is the short-term one that limits the peak interference power (PIP) at each of the fading states. Clearly, the PIP constraint is more restrictive than the AIP counterpart given the same average and peak interference-power threshold. From the CR's perspective, the AIP constraint is more favorable than the PIP, since the former provides the CR more flexibility for dynamically allocating transmit powers over the fading states and, thus, achieves larger fading channel capacities [7], [8]. However, the effect of the AIP- and PIP-based CR power control on the PR transmissions has not yet been studied in the literature, to the author's best knowledge. At a first glance, the more restrictive PIP constraint seems to be a better option than the AIP from the perspective of protecting the PR. Some surprisingly, in this paper the contrary conclusion is rigourously shown, i.e., the AIP constraint is indeed superior over the PIP in terms of various forms of capacity limits achievable for the PR fading channel, e.g., the ergodic and outage capacities. This result is due to an interesting interference diversity phenomenon for the PR transmission: Due to the convexity of the capacity function with respect to the noise/interferecne power, more randomized interference powers over the fading states at the PR receiver in the AIP case are more advantageous over deterministic ones in the PIP case for minimizing the resultant PR capacity losses. Therefore, this paper provides an important design rule for the CR networks in practice, i.e., the AIP constraint may result in improved fading channel capacities over the PIP for both the CR and PR transmissions.

The rest of this paper is organized as follows. Section II presents the system model for spectrum sharing. Section $\amalg$ considers the CR link and summarizes the results known in the literature on the CR fading channel capacities and the corresponding optimal power-control policies under the AIP or the PIP constraint. Section IV then studies various forms of the PR fading channel capacities under the interference from the CR transmitter due to the AIP- or PIP-based CR power control, and proves that the AIP constraint results in larger channel capacities than the PIP for the same power threshold. Section $\mathrm{V}$ considers both PR and CR transmissions and shows the simulation results on their jointly 


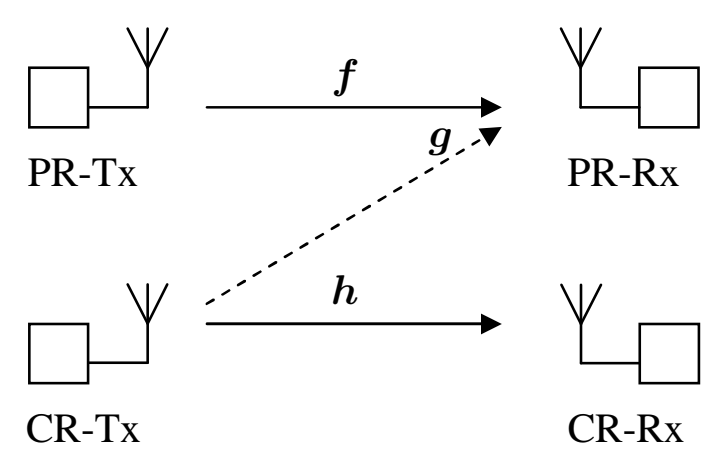

Fig. 1. Spectrum sharing between a PR link and a CR link.

achievable capacities under spectrum sharing. Finally, Section VI concludes this paper.

Notation: $|z|$ denotes the Euclidean norm of a complex number $z . \mathbb{E}[\cdot]$ denotes the statistical expectation. $\operatorname{Pr}\{\cdot\}$ denotes the probability. $\mathbf{1}(\mathcal{A})$ denotes the indicator function taking the value of one if the event $\mathcal{A}$ is true, and the value of zero otherwise. The distribution of a circular symmetric complex Gaussian (CSCG) random variable (r.v.) with mean $x$ and variance $y$ is denoted as $\mathcal{C N}(x, y)$, and $\sim$ means "distributed as". $\max (x, y)$ and $\min (x, y)$ denote, respectively, the maximum and the minimum between two real numbers $x$ and $y$; for a real number $a,(a)^{+} \triangleq \max (0, a)$.

\section{SySTEM MODEL}

As shown in Fig. 1, a spectrum sharing scenario is considered where a CR link consisting of a CR transmitter (CR-Tx) and a CR receiver (CR-Rx) shares the same bandwidth for transmission with an existing PR link consisting of a PR transmitter (PR-Tx) and a PR receiver (PR-Rx). All terminals are assumed to be equipped with a single antenna. We consider a slow-fading environment and, for simplicity, assume a block-fading (BF) channel model for all the channels involved in the PR-CR network. Furthermore, we assume coherent communication and thus only the fading channel power gain (amplitude square) is of interest. Denote $\boldsymbol{h}$ as the r.v. for the power gain of the fading channel from CR-Tx to CR-Rx. Similarly, $g$ and $f$ are defined for the fading channel from CR-Tx to PR-Rx and PR-Tx to PR-Rx, respectively. For convenience, in this paper we ignore the channel from PR-Tx to CR-Rx. Denote $i$ as the joint fading state for all the channels involved. Then, let $h_{i}$ be the $i$ th component in $\boldsymbol{h}$ for fading state $i$; similarly, $g_{i}$ and $f_{i}$ are defined. It is assumed that $h_{i}, g_{i}$, and $f_{i}$ are independent 
of each other, and all of them have continuous probability density functions (PDFs). It is also assumed that the additive noises at both PR-Rx and CR-Rx are independent CSCG r.v.s each $\sim \mathcal{C N}(0,1)$. Since we are interested in the information-theoretic limits of the PR and CR channels, it is assumed that the optimal Gaussian codebook is used at both PR-Tx and CR-Tx.

For the PR link, the transmit power at fading state $i$ is denoted as $q_{i}$. It is assumed that the PR is oblivious to the $\mathrm{CR}$ transmission and thus does not attempt to protect the $\mathrm{CR}$ nor cooperate with the CR for transmission. Due to the CR transmission, PR-Rx may observe an additional interference power, denoted as $I_{i}=g_{i} p_{i}$, at fading state $i$ where $p_{i}$ denotes the CR transmit power at fading state $i$. The PR power-control policy, denoted as $\mathcal{P}_{\mathrm{PR}}(\boldsymbol{f}, \boldsymbol{I})$, is in general a mapping from $f_{i}$ and $I_{i}$ to $q_{i}$ for each $i$, with $I_{i}$ being the $i$ th component of $\boldsymbol{I}$, subject to an average transmit power constraint $Q$, i.e., $\mathbb{E}\left[q_{i}\right] \leq Q$. By treating the interference from CR-Tx as the additional Gaussian noise at PR-Rx, the mutual information of the PR fading channel for fading state $i$ under a given $\mathcal{P}_{\mathrm{PR}}(\boldsymbol{f}, \boldsymbol{I})$ can then be expressed as [14]

$$
R_{\mathrm{PR}}(i)=\log \left(1+\frac{f_{i} q_{i}}{1+I_{i}}\right) .
$$

For the CR link, since the CR needs to protect the PR transmission, the CR power-control policy needs to be aware of both the PR and CR transmissions. It is assumed that the channel power gains $g_{i}$ and $h_{i}$ are perfectly known at CR-Tx for each $\left.i\right]_{1}$ Thus, the CR power-control policy can be expressed as $\mathcal{P}_{\mathrm{CR}}(\boldsymbol{h}, \boldsymbol{g})$ with $\boldsymbol{h}$ consisting of $h_{i}$ 's, subject to an average transmit power constraint $P$, i.e., $\mathbb{E}\left[p_{i}\right] \leq P$. The mutual information of the CR fading channel for fading state $i$ under a given $\mathcal{P}_{\mathrm{CR}}(\boldsymbol{h}, \boldsymbol{g})$ can then be expressed as

$$
R_{\mathrm{CR}}(i)=\log \left(1+h_{i} p_{i}\right)
$$

In this paper, we assume that the $\mathrm{CR}$ protects the $\mathrm{PR}$ transmission via transmit power control by applying the interference-power constraint at PR-Rx, in the form of either the AIP or the PIP. The AIP constraint regulates the average interference power at PR-Rx over all the fading states and is thus expressed as

$$
\mathbb{E}\left[I_{i}\right] \leq \Gamma_{a} \text { or } \mathbb{E}\left[g_{i} p_{i}\right] \leq \Gamma_{a}
$$

\footnotetext{
${ }^{1}$ In practice, the channel power gain between CR-Tx and PR-Rx can be obtained at CR-Tx via, e.g., estimating the received signal power from PR-Rx when it transmits, under the assumptions of the pre-knowledge on the PR-Rx transmit power level and the channel reciprocity.
} 
where $\Gamma_{a}$ denotes the predefined AIP threshold. In contrast, the PIP constraint limits the peak interference power at PR-Rx at each of the fading states and is thus expressed as

$$
I_{i} \leq \Gamma_{p}, \forall i \text { or } g_{i} p_{i} \leq \Gamma_{p}, \forall i
$$

where $\Gamma_{p}$ denotes the predefined PIP threshold. Note that the PIP constraint is in general more restrictive over the AIP. This can be easily seen by observing that given $\Gamma_{p}=\Gamma_{a}$, (4) implies (3) but not vice versa. Therefore, from the CR's perspective, applying the AIP constraint is more favorable than the PIP because the former provides the CR more flexibility for adapting transmit powers over the fading states.

In this paper, we consider two well-known capacity limits for the fading PR and CR channels, namely, the ergodic capacity and the outage capacity. The ergodic capacity measures the maximum average rate over the fading states [15], while the resultant mutual information for each fading state can be variable. In contrast, the outage capacity measures the maximum constant rate that is achievable over each of the fading states with a guaranteed outage probability [16], [17]. In the extreme case of zero outage probability, the outage capacity is also known as the delay-limited capacity [18]. In general, the ergodic and delay-limited capacities can be considered as the throughput limits for a fading channel with no and with minimal transmission delay requirement, respectively.

\section{CR CAPACITIES UNDER AIP VERSUS PIP CONSTRAINT}

In this section, we summarize the results known in the literature on the CR fading channel capacities and the corresponding optimal power-control policies under the AIP or the PIP constraint. Consider first the AIP case. The optimal $\mathcal{P}_{\mathrm{CR}}(\boldsymbol{h}, \boldsymbol{g})$ to achieve the ergodic capacity of the CR fading channel is expressed as [6]

$$
p_{i}^{\mathrm{ER}, a}=\left(\frac{1}{\nu g_{i}}-\frac{1}{h_{i}}\right)^{+}
$$

where $\nu$ is a positive constant determined from $\mathbb{E}\left[g_{i} p_{i}^{\mathrm{ER}, a}\right]=\Gamma_{a}$. Note that the above power control resembles the well-known "water filling (WF)" power control [14], [15], which achieves the ergodic capacity of the conventional fading channel, whereas there is also a key difference here: In (5), the so-called "water level" for WF, $1 /\left(\nu g_{i}\right)$, depends on the channel power gain $g_{i}$ from CR-Tx to PR-Rx as compared to being a constant in the standard WF power control. Substituting (5) into $R_{\mathrm{CR}}(i)$ given 
in (2) and taking the expectation of the resultant $R_{\mathrm{CR}}(i)$ over $i$, we obtain the ergodic capacity for the CR under the AIP constraint, denoted as $C_{\mathrm{CR}}^{\mathrm{ER}, a}$. On the other hand, the optimal $\mathcal{P}_{\mathrm{CR}}(\boldsymbol{h}, \boldsymbol{g})$ to achieve the outage capacity of the $\mathrm{CR}$ fading channel with a guaranteed outage probability, $\epsilon_{0}$, is expressed as [7], [8]

$$
p_{i}^{\mathrm{OUT}, a}= \begin{cases}\frac{\zeta_{a}}{h_{i}}, & \frac{h_{i}}{g_{i}} \geq \lambda \\ 0, & \text { otherwise }\end{cases}
$$

where $\lambda$ is a nonnegative constant determined from $\operatorname{Pr}\left\{h_{i} / g_{i}<\lambda\right\}=\epsilon_{0}$, and $\zeta_{a}$ is the constant signalto-noise ratio $(\mathrm{SNR})$ at $\mathrm{CR}-\mathrm{Rx}$ obtained from $\mathbb{E}\left[g_{i} p_{i}^{\mathrm{OUT}, a}\right]=\Gamma_{a}$. Note that the above power control resembles the well-known "truncated channel inversion (TCI)" power control [15] to achieve the outage capacity of the conventional fading channel [17], while there is also a difference between (6) and the standard TCI on the threshold value $\lambda$ for power truncation (no transmission): in (6) $\lambda$ depends on the ratio between $h_{i}$ and $g_{i}$, as compared to only $h_{i}$ in the standard TCI. The corresponding outage capacity, denoted as $C_{\mathrm{CR}}^{\mathrm{OUT}, a}\left(\epsilon_{0}\right)$, is then obtained as $\log \left(1+\zeta_{a}\right)$. Note that if $\epsilon_{0}=0$, it then follows that $\lambda=0$ and the resultant power-control policy in (6) becomes the "channel inversion (CI)" power control [15], which achieves the delay-limited capacity for the CR [17], denoted as $C_{\mathrm{CR}}^{\mathrm{DL}, a}$.

Consider next the PIP case. It is easy to show that in this case the optimal $\mathcal{P}_{\mathrm{CR}}(\boldsymbol{h}, \boldsymbol{g})$ should use the maximum possible transmit power for each fading state $i$ so as to maximize both the ergodic and the outage capacities. 2 thus, we have

$$
p_{i}^{\mathrm{ER}, p}=p_{i}^{\mathrm{OUT}, p}=\frac{\Gamma_{p}}{g_{i}}, \forall i
$$

The resultant ergodic capacity, denoted as $C_{\mathrm{CR}}^{\mathrm{ER}, p}$, is then obtained accordingly from (2). The resultant outage probability, $\epsilon_{0}$, can be shown equal to $\operatorname{Pr}\left\{\left(\Gamma_{p} / g_{i}\right) h_{i}<\zeta_{p}\right\}$ where $\zeta_{p}$ is the constant SNR at CR-Rx. For a given $\epsilon_{0}$, the corresponding $\zeta_{p}$ can thus be obtained, as well as the corresponding outage capacity, $C_{\mathrm{CR}}^{\mathrm{OUT}, p}\left(\epsilon_{0}\right)=\log \left(1+\zeta_{p}\right)$. It is easy to see that if $\epsilon_{0}=0$, it follows that $\zeta_{p}=0$ and thus the delay-limited capacity for the CR under the PIP constraint, denoted as $C_{\mathrm{CR}}^{\mathrm{DL}, p}$, is always zero.

\footnotetext{
${ }^{2}$ It is noted that to achieve the same outage capacity for the CR, under the assumption that the CR channel power gain $h_{i}$ is known at CR-Tx for each $i$, it is possible for the CR power control to assign a smaller power value $\zeta_{p} / h_{i}$ than $\Gamma_{p} / g_{i}$ if the former happens to be smaller than the latter for some $i$. However, if $h_{i}$ s are not available at CR-Tx, it is optimal for the CR to assign the maximum possible transmit power $\Gamma_{p} / g_{i}$ for each $i$ to minimize the outage probability. Therefore, in this paper we consider that $p_{i}^{\text {OUT, } p}=\Gamma_{p} / g_{i}, \forall i$.
} 
Comparing the power allocations in (5) and (6) for the AIP case with those in (7) for the PIP case, it is easy to see that the former power allocations are more flexible than the latter ones over the fading states. Furthermore, the AIP-based power control depends on both the channel power gains, $h_{i}$ and $g_{i}$, while the PIP-based power control only depends on $g_{i}$. As a result, under the same average and peak power threshold, i.e., $\Gamma_{a}=\Gamma_{p}$, it is easy to show that $C_{\mathrm{CR}}^{\mathrm{ER}, a} \geq C_{\mathrm{CR}}^{\mathrm{ER}, p}, C_{\mathrm{CR}}^{\mathrm{OUT}, a}\left(\epsilon_{0}\right) \geq C_{\mathrm{CR}}^{\mathrm{OUT}, p}\left(\epsilon_{0}\right)$, and $C_{\mathrm{CR}}^{\mathrm{DL}, a} \geq C_{\mathrm{CR}}^{\mathrm{DL}, p}$. Thus, the AIP is superior over the PIP in terms of the fading channel capacity limits achievable for the CR.

\section{PR CAPACITIES UNDER AIP VERSUS PIP CONSTRAINT}

In this section, we will present the main contributions of this paper on the comparison of the effects of AIP and PIP constraints on various fading channel capacities for the PR. For fair comparison, we consider the same average and peak interference-power threshold, i.e., $\Gamma_{a}=\Gamma_{p}=\Gamma$. Note that both AIP and PIP constraints are satisfied with equalities at PR-Rx for all the CR power-control policies presented in Section $\amalg$ III, i.e., for the AIP case, $\mathbb{E}\left[I_{i}\right]=\Gamma$; and for the PIP case, $I_{i}=\Gamma, \forall i$. In the following two subsections, we consider the ergodic capacity and the outage capacity for the PR fading channel, respectively.

\section{A. Ergodic Capacity}

1) Constant-Power Policy: The simplest power control for the PR is the constant-power (CP) policy, i.e.,

$$
q_{i}^{\mathrm{CP}}=Q, \forall i
$$

$\mathrm{CP}$ is an attractive scheme in practice from an implementation viewpoint since it does not require any CSI on the PR fading channel at PR-Tx. In addition, CP satisfies a peak transmit-power constraint for all the fading states. With CP, the ergodic capacity of the PR fading channel in the AIP case can be obtained from (11) and expressed as

$$
C_{\mathrm{PR}, \mathrm{CP}}^{\mathrm{ER}, a}=\mathbb{E}\left[\log \left(1+\frac{f_{i} Q}{1+I_{i}}\right)\right]
$$

and in the PIP case expressed as

$$
C_{\mathrm{PR}, \mathrm{CP}}^{\mathrm{ER}, p}=\mathbb{E}\left[\log \left(1+\frac{f_{i} Q}{1+\Gamma}\right)\right] .
$$


Theorem 4.1: With the CP policy for the PR, $C_{\mathrm{PR}, \mathrm{CP}}^{\mathrm{ER}, a} \geq C_{\mathrm{PR}, \mathrm{CP}}^{\mathrm{ER}, p}$, under the same average and peak power threshold $\Gamma$.

Proof: The following equalities/inequality hold:

$$
\begin{aligned}
C_{\mathrm{PR}, \mathrm{CP}}^{\mathrm{ER}, a} & \stackrel{(a)}{=} \mathbb{E}_{f} \mathbb{E}_{I}\left[\log \left(1+\frac{f_{i} Q}{1+I_{i}}\right)\right] \\
& \stackrel{(b)}{\geq} \mathbb{E}_{f}\left[\log \left(1+\frac{f_{i} Q}{1+\mathbb{E}\left[I_{i}\right]}\right)\right] \\
& \stackrel{(c)}{=} \mathbb{E}_{f}\left[\log \left(1+\frac{f_{i} Q}{1+\Gamma}\right)\right] \\
& \stackrel{(d)}{=} C_{\mathrm{PR}, \mathrm{CP}}^{\mathrm{ER}, p}
\end{aligned}
$$

where $(a)$ is from (9) and due to independence of $f_{i}$ and $g_{i}$ and thus $f_{i}$ and $I_{i}$; $(b)$ is due to convexity of the function $f(x)=\log \left(1+\frac{\kappa}{1+x}\right)$ where $\kappa$ is any positive constant and $x \geq 0$, and Jensen's inequality (e.g., [14]); $(c)$ and $(d)$ are due to $\mathbb{E}\left[I_{i}\right]=\Gamma$ and (10), respectively.

Theorem 4.1 suggests that, some surprisingly, the AIP constraint that results in randomized interference power levels over the fading states at PR-Rx is in fact more advantageous for improving the PR ergodic capacity over the PIP constraint that results in constant interference power levels at all the fading states, for the same value of $\Gamma$. As shown in the above proof, this result is mainly due to the convexity of the capacity function with respect to the noise/interference power. We thus name this interesting phenomenon for the PR transmission in a CR network as "interference diversity".

2) Water-Filling Power Control: If the effective channel power gain, $f_{i} /\left(1+I_{i}\right)$, for the PR fading channel is known at PR-Tx for each $i$, the optimal $\mathcal{P}_{\mathrm{PR}}(\boldsymbol{f}, \boldsymbol{I})$ to achieve the ergodic capacity for the PR is the standard WF power-control policy. In the AIP case, the optimal power allocation is expressed as

$$
q_{i}^{\mathrm{WF}, a}=\left(\frac{1}{\mu_{a}}-\frac{1+I_{i}}{f_{i}}\right)^{+}
$$

where $\mu_{a}$ controls the water level, $1 / \mu_{a}$, with which $\mathbb{E}\left[q_{i}^{\mathrm{WF}, a}\right]=Q$. From (11), the ergodic capacity for the PR in the AIP case is obtained as

$$
C_{\mathrm{PR}, \mathrm{WF}}^{\mathrm{ER}, a}=\mathbb{E}\left[\left(\log \left(\frac{f_{i}}{\mu_{a}\left(1+I_{i}\right)}\right)\right)^{+}\right]
$$


Similarly, we can obtain the optimal WF-based power control for the PR in the PIP case as

$$
q_{i}^{\mathrm{WF}, p}=\left(\frac{1}{\mu_{p}}-\frac{1+\Gamma}{f_{i}}\right)^{+}
$$

where $\mu_{p}$ is obtained from $\mathbb{E}\left[q_{i}^{\mathrm{WF}, p}\right]=Q$. The corresponding ergodic capacity then becomes

$$
C_{\mathrm{PR}, \mathrm{WF}}^{\mathrm{ER}, p}=\mathbb{E}\left[\left(\log \left(\frac{f_{i}}{\mu_{p}(1+\Gamma)}\right)\right)^{+}\right] .
$$

Next, we first show that an intuitive method to compare $C_{\mathrm{PR}, \mathrm{WF}}^{\mathrm{ER}, a}$ in (12) and $C_{\mathrm{PR}, \mathrm{WF}}^{\mathrm{ER}, p}$ in (14) does not work here. Then, we present a different method for such comparison. One intuitive method to compare $C_{\mathrm{PR}, \mathrm{WF}}^{\mathrm{ER}, a}$ and $C_{\mathrm{PR}, \mathrm{WF}}^{\mathrm{ER} p}$ would be as follows. If it can be shown that $\mu_{a}<\mu_{p}$, then due to convexity of the function $g(x)=\left(\log \left(\frac{\kappa}{1+x}\right)\right)^{+}$where $\kappa$ is a positive constant and $x \geq 0$, and similarly like the proof of Theorem 4.1, it can be shown that $C_{\mathrm{PR}, \mathrm{WF}}^{\mathrm{ER}, a}>C_{\mathrm{PR}, \mathrm{WF}}^{\mathrm{ER}, p}$. Unfortunately, in the following we prove by contradiction that the opposite inequality is in fact true for $\mu_{a}$ and $\mu_{p}$. Thus, we can not conclude which one of $C_{\mathrm{PR}, W F}^{\mathrm{ER}, a}$ and $C_{\mathrm{PR}, W F}^{\mathrm{ER}, p}$ is indeed larger by this intuitive method.

Supposing that $\mu_{a}<\mu_{p}$, we then have

$$
\begin{aligned}
\mathbb{E}\left[q_{i}^{\mathrm{WF}, a}\right] & =\mathbb{E}\left[\left(\frac{1}{\mu_{a}}-\frac{1+I_{i}}{f_{i}}\right)^{+}\right] \\
& >\mathbb{E}\left[\left(\frac{1}{\mu_{p}}-\frac{1+I_{i}}{f_{i}}\right)^{+}\right] \\
& =\mathbb{E}_{f} \mathbb{E}_{I}\left[\left(\frac{1}{\mu_{p}}-\frac{1+I_{i}}{f_{i}}\right)^{+}\right] \\
& \stackrel{(a)}{\geq} \mathbb{E}_{f}\left[\left(\frac{1}{\mu_{p}}-\frac{1+\mathbb{E}\left[I_{i}\right]}{f_{i}}\right)^{+}\right] \\
& =\mathbb{E}\left[\left(\frac{1}{\mu_{p}}-\frac{1+\Gamma}{f_{i}}\right)^{+}\right] \\
& =\mathbb{E}\left[q_{i}^{\mathrm{WF}, p}\right] \\
& =Q
\end{aligned}
$$

where $(a)$ is due to convexity of the function $z(x)=\left(\kappa_{1}-\frac{1+x}{\kappa_{2}}\right)^{+}$where $\kappa_{1}$ and $\kappa_{2}$ are positive constants and $x \geq 0$, and Jensen's inequality. Since it is known that $\mathbb{E}\left[q_{i}^{\mathrm{WF}, a}\right]=Q$, which contradicts with $\mathbb{E}\left[q_{i}^{\mathrm{WF}, a}\right]>Q$ shown in the above under the presumption that $\mu_{a}<\mu_{p}$, it thus concludes that $\mu_{a} \geq \mu_{p}$ 
From the above discussions, we know that an alternative approach is needed for comparing $C_{\mathrm{PR}, \mathrm{WF}}^{\mathrm{ER}, a}$ and $C_{\mathrm{PR}, \mathrm{WF}}^{\mathrm{ER},}$. The result for this comparison and its proof are given below:

Theorem 4.2: With the WF power control for the $\mathrm{PR}, C_{\mathrm{PR}, \mathrm{WF}}^{\mathrm{ER}, a} \geq C_{\mathrm{PR}, \mathrm{WF}}^{\mathrm{ER}, p}$, under the same average and peak power threshold $\Gamma$.

Proof: The proof is based on the Lagrange duality of convex optimization [19]. First, we rewrite $C_{\mathrm{PR}, \mathrm{WF}}^{\mathrm{ER}, a}$ and $C_{\mathrm{PR}, \mathrm{WF}}^{\mathrm{ER}, p}$ as the optimal values of the following min-max optimization problems:

$$
C_{\mathrm{PR}, \mathrm{WF}}^{\mathrm{ER}, a}=\min _{\mu: \mu \geq 0} \max _{\left\{q_{i}\right\}: q_{i} \geq 0, \forall i} \mathbb{E}\left[\log \left(1+\frac{f_{i} q_{i}}{1+I_{i}}\right)\right]-\mu\left(\mathbb{E}\left[q_{i}\right]-Q\right)
$$

and

$$
C_{\mathrm{PR}, \mathrm{WF}}^{\mathrm{ER}, p}=\min _{\mu: \mu \geq 0} \max _{\left\{q_{i}\right\}: q_{i} \geq 0, \forall i} \mathbb{E}\left[\log \left(1+\frac{f_{i} q_{i}}{1+\Gamma}\right)\right]-\mu\left(\mathbb{E}\left[q_{i}\right]-Q\right),
$$

respectively. Note that $\mu_{a}$ and $\left\{q_{i}^{\mathrm{WF}, a}\right\}$ are the optimal solutions to the "min" and "max" problems in (15), respectively, and $\mu_{p}$ and $\left\{q_{i}^{\mathrm{WF}, p}\right\}$ are the optimal solutions to the "min" and "max" problems in (16), respectively. Then, we have the following equalities/inequalities:

$$
\begin{aligned}
C_{\mathrm{PR}, \mathrm{WF}}^{\mathrm{ER}, p} & =\min _{\mu: \mu \geq 0} \mathbb{E}\left[\left(\log \left(\frac{f_{i}}{(1+\Gamma) \mu}\right)\right)^{+}\right]-\mathbb{E}\left[\left(1-\frac{(1+\Gamma) \mu}{f_{i}}\right)^{+}\right]+\mu Q \\
& \leq \mathbb{E}\left[\left(\log \left(\frac{f_{i}}{(1+\Gamma) \mu_{a}}\right)\right)^{+}\right]-\mathbb{E}\left[\left(1-\frac{(1+\Gamma) \mu_{a}}{f_{i}}\right)^{+}\right]+\mu_{a} Q \\
& =\mathbb{E}_{f}\left[\left(\log \left(\frac{f_{i}}{\left(1+\mathbb{E}\left[I_{i}\right]\right) \mu_{a}}\right)\right)^{+}-\left(1-\frac{\left(1+\mathbb{E}\left[I_{i}\right]\right) \mu_{a}}{f_{i}}\right)^{+}\right]+\mu_{a} Q \\
& \leq \mathbb{E}_{f} \mathbb{E}_{I}\left[\left(\log \left(\frac{f_{i}}{\left(1+I_{i}\right) \mu_{a}}\right)\right)^{+}-\left(1-\frac{\left(1+I_{i}\right) \mu_{a}}{f_{i}}\right)^{+}\right]+\mu_{a} Q \\
& =\min _{\mu: \mu \geq 0} \mathbb{E}\left[\left(\log \left(\frac{f_{i}}{\left(1+I_{i}\right) \mu}\right)\right)^{+}\right]-\mathbb{E}\left[\left(1-\frac{\left(1+I_{i}\right) \mu}{f_{i}}\right)^{+}\right]+\mu Q \\
& =C_{\mathrm{PR}, \mathrm{WF}}^{\mathrm{ER}, a}
\end{aligned}
$$

where (17) is obtained by substituting $\left\{q_{i}^{\mathrm{WF}, p}\right\}$ in (13) with $\mu_{p}$ replaced by an arbitrary positive $\mu$ into (16); (18) is due to the fact that $\mu_{a}$ is not the minimizer $\mu_{p}$ for $(17) ;(19)$ is due to $\mathbb{E}\left[I_{i}\right]=\Gamma$; (20) is due to convexity of the function in $\mathbb{E}_{f}[\cdot]$ of $(19)$ with respect to $\mathbb{E}\left[I_{i}\right]$ for any given $f_{i}$ and Jensen's inequality; (21) and (22) are due to the fact that $\mu_{a}$ and $\left\{q_{i}^{\mathrm{WF}, a}\right\}$ in (11) are the optimal solutions to the min-max optimization problem in (15). 
Theorem 4.2 suggests that, similarly like the CP policy, under the WF-based power control, randomized interference power levels due to the CR transmission in the AIP case is superior over constant interference power levels in the PIP case in terms of the maximum achievable PR ergodic capacity. However, the interference diversity gain observed here is not as obvious as that in the CP case due to the more complex WF-based PR power control.

\section{B. Outage Capacity}

1) Constant-Power Policy: With the CP policy in (8), for a given outage probability, $\epsilon_{0}$, the maximum achievable constant SNR at PR-Rx, denoted as $\gamma_{a}$, in the AIP case can be obtained from $\operatorname{Pr}\left\{\left(f_{i} Q\right) /(1+\right.$ $\left.\left.I_{i}\right)<\gamma_{a}\right\}=\epsilon_{0}$, and the corresponding outage capacity, denoted as $C_{\mathrm{PR}, \mathrm{CP}}^{\mathrm{OUT}, a}\left(\epsilon_{0}\right)$, is equal to $\log \left(1+\gamma_{a}\right)$. Similarly, for the same $\epsilon_{0}$, the maximum achievable constant SNR at PR-Rx, $\gamma_{p}$, in the PIP case can be obtained from $\operatorname{Pr}\left\{\left(f_{i} Q\right) /(1+\Gamma)<\gamma_{p}\right\}=\epsilon_{0}$, and the corresponding outage capacity, $C_{\mathrm{PR}, \mathrm{CP}}^{\mathrm{OUT}}\left(\epsilon_{0}\right)$, is obtained as $\log \left(1+\gamma_{p}\right)$.

Instead of comparing $C_{\mathrm{PR}, \mathrm{CP}}^{\mathrm{OUT}, a}\left(\epsilon_{0}\right)$ and $C_{\mathrm{PR}, \mathrm{CP}}^{\mathrm{OUT}, p}\left(\epsilon_{0}\right)$ directly, we consider the following equivalent problem: Supposing that $\gamma_{a}=\gamma_{p}=\gamma_{0}$, we compare the resultant minimum outage probabilities, denoted as $\epsilon_{a}$ and $\epsilon_{p}$ in the AIP and PIP cases, respectively. If $\epsilon_{a} \leq \epsilon_{p}$ for any given $\gamma_{0}$, we conclude that $C_{\mathrm{PR}, \mathrm{CP}}^{\mathrm{OUT}, a}\left(\epsilon_{0}\right) \geq C_{\mathrm{PR}, \mathrm{CP}}^{\mathrm{OUT}, p}\left(\epsilon_{0}\right)$ for any $\epsilon_{0}$. This is true because if $\epsilon_{a} \leq \epsilon_{p}$, we can increase $\gamma_{a}$ above $\gamma_{0}$ so that $\epsilon_{a}$ increases until it becomes equal to $\epsilon_{p}$; since $\gamma_{a} \geq \gamma_{0} \geq \gamma_{p}$, it follows that $C_{\mathrm{PR}, \mathrm{CP}}^{\mathrm{OUT}, a}\left(\epsilon_{p}\right) \geq C_{\mathrm{PR}, \mathrm{CP}}^{\mathrm{OUT}, p}\left(\epsilon_{p}\right)$. Similarly, if $\epsilon_{a} \geq \epsilon_{p}$ for any given $\gamma_{0}$, we conclude that $C_{\mathrm{PR}, \mathrm{CP}}^{\mathrm{OUT}, a}\left(\epsilon_{0}\right) \leq C_{\mathrm{PR}, \mathrm{CP}}^{\mathrm{OUT}, p}\left(\epsilon_{0}\right)$ for any $\epsilon_{0}$.

To compare $\epsilon_{a}$ and $\epsilon_{p}$ for the same given $\gamma_{0}$, we first express $\epsilon_{a}$ as

$$
\begin{aligned}
\epsilon_{a} & =\operatorname{Pr}\left\{\frac{f_{i} Q}{1+I_{i}}<\gamma_{0}\right\} \\
& =\mathbb{E}_{I}\left[\mathbb{E}_{f}\left[\mathbf{1}\left(\frac{f_{i} Q}{1+I_{i}}<\gamma_{0}\right)\right]\right] \\
& =\mathbb{E}_{I}\left[G_{f}\left(\frac{\left(1+I_{i}\right) \gamma_{0}}{Q}\right)\right]
\end{aligned}
$$

where $G_{f}(x)$ is the cumulative density function (CDF) for $\boldsymbol{f}$, i.e., $G_{f}(x)=\operatorname{Pr}\{\boldsymbol{f}<x\}$. Similarly, we can express $\epsilon_{p}$ as

$$
\epsilon_{p}=G_{f}\left(\frac{(1+\Gamma) \gamma_{0}}{Q}\right)
$$


By Jensen's inequality, from (25) and (26), it follows that $\epsilon_{a} \leq \epsilon_{p}$ if $G_{f}(x)$ is a convex function. Similarly, $\epsilon_{a} \geq \epsilon_{p}$ if $G_{f}(x)$ is a concave function. We thus have the following theorem:

Theorem 4.3: With the CP policy for the PR, $C_{\mathrm{PR}, \mathrm{CP}}^{\mathrm{OUT}, a}\left(\epsilon_{0}\right) \geq C_{\mathrm{PR}, \mathrm{CP}}^{\mathrm{OUT}, p}\left(\epsilon_{0}\right), \forall \epsilon_{0}$, under the same average and peak power threshold $\Gamma$, if $G_{f}(x)$ is a convex function; and $C_{\mathrm{PR}, \mathrm{CP}}^{\mathrm{OUT}, a}\left(\epsilon_{0}\right) \leq C_{\mathrm{PR}, \mathrm{CP}}^{\mathrm{OUT}, p}\left(\epsilon_{0}\right), \forall \epsilon_{0}$, if $G_{f}(x)$ is a concave function.

Theorem 4.3 suggests that for the CP policy, whether the AIP or the PIP constraint results in a larger PR outage capacity depends on the convexity/concavity of the CDF of the PR fading channel power gain. As an example, for the standard Rayleigh fading model, it is known that $G_{f}(x)$ has an exponential distribution that is convex and, thus, $C_{\mathrm{PR}, \mathrm{CP}}^{\mathrm{OUT}, a}\left(\epsilon_{0}\right) \geq C_{\mathrm{PR}, \mathrm{CP}}^{\mathrm{OUT}, p}\left(\epsilon_{0}\right)$. However, in general, whether the interference diversity gain is present depends on the PR fading channel distribution.

2) Channel-Inversion Power Control: Next, we consider the special case of the CR outage capacity with zero outage probability, i.e., the delay-limited capacity, which is achievable by the CI power-control policy. In the AIP case, the optimal PR power allocation is expressed as

$$
q_{i}^{\mathrm{CI}, a}=\frac{\gamma_{a}\left(1+I_{i}\right)}{f_{i}}
$$

and in the PIP case expressed as

$$
q_{i}^{\mathrm{CI}, p}=\frac{\gamma_{p}(1+\Gamma)}{f_{i}}
$$

where $\gamma_{a}$ and $\gamma_{p}$ are the constant SNRs at PR-Rx for the AIP and PIP cases, respectively. Given $\mathbb{E}\left[q_{i}\right]=Q, \gamma_{a}$ and $\gamma_{p}$ can be obtained from (27) and (28) as

$$
\gamma_{a}=\frac{Q}{\mathbb{E}\left[\frac{1+I_{i}}{f_{i}}\right]}
$$

and

$$
\gamma_{p}=\frac{Q}{(1+\Gamma) \mathbb{E}\left[\frac{1}{f_{i}}\right]},
$$

respectively. Since $f_{i}$ is independent of $I_{i}$, we have

$$
\mathbb{E}\left[\frac{1+I_{i}}{f_{i}}\right]=\mathbb{E}_{f}\left[\frac{1+\mathbb{E}\left[I_{i}\right]}{f_{i}}\right]=(1+\Gamma) \mathbb{E}\left[\frac{1}{f_{i}}\right]
$$

and thus it follows from (29) and (30) that $\gamma_{a}=\gamma_{p}$. Hence, we conclude that the PR delay-limited capacities, expressed as $C_{\mathrm{PR}, a}^{\mathrm{DL}}=\log \left(1+\gamma_{a}\right)$ and $C_{\mathrm{PR}}^{\mathrm{DL}, p}=\log \left(1+\gamma_{p}\right)$, for the AIP and PIP cases, respectively, are indeed identical. The following theorem thus holds: 
Theorem 4.4: With the CI power control for the $\mathrm{PR}, C_{\mathrm{PR}}^{\mathrm{DL}, a}=C_{\mathrm{PR}}^{\mathrm{DL}, p}$, under the same average and peak power threshold $\Gamma$.

Theorem 4.4 suggests that for the CI power control, the loss of the PR delay-limited capacity due to randomized interference powers from CR-Tx is identical to that due to constant interference powers, i.e., the AIP constraint is at least no worse than the PIP from the PR's perspective of delivering zero-delay and constant-rate data traffic.

3) Truncated-Channel-Inversion Power Control: Lastly, we consider the general outage capacity for the PR achievable by the TCI power-control policy. In the AIP case, the optimal TCI power control is expressed as

$$
q_{i}^{\mathrm{TCI}, a}= \begin{cases}\frac{\gamma_{a}\left(1+I_{i}\right)}{f_{i}}, & \frac{f_{i}}{1+I_{i}} \geq \theta_{a} \\ 0, & \text { otherwise }\end{cases}
$$

where $\theta_{a}$ is the threshold for the effective channel power gain above which CI power control is applied to achieve a constant receiver SNR, $\gamma_{a}$, and below which no transmission is implemented. Similarly, the TCI power control in the PIP case is expressed as

$$
q_{i}^{\mathrm{TCI}, p}= \begin{cases}\frac{\gamma_{p}(1+\Gamma)}{f_{i}}, & \frac{f_{i}}{1+\Gamma} \geq \theta_{p} \\ 0, & \text { otherwise }\end{cases}
$$

where $\theta_{p}$ is the threshold for power truncation. Given the outage probability $\epsilon_{0}, \theta_{a}$ and $\theta_{p}$ can be obtained from $\operatorname{Pr}\left\{f_{i} /\left(1+I_{i}\right)<\theta_{a}\right\}=\epsilon_{0}$ and $\operatorname{Pr}\left\{f_{i} /(1+\Gamma)<\theta_{p}\right\}=\epsilon_{0}$, respectively. Then, $\gamma_{a}$ and $\gamma_{p}$ can be obtained from $\mathbb{E}\left[q_{i}^{\mathrm{TCI}, a}\right]=Q$ and $\mathbb{E}\left[q_{i}^{\mathrm{TCI}, p}\right]=Q$, respectively. The corresponding outage capacities for the PR, denoted as $C_{\mathrm{PR}, \mathrm{TCI}}^{\mathrm{OUT}, a}\left(\epsilon_{0}\right)$ and $C_{\mathrm{PR}, \mathrm{TCI}}^{\mathrm{OUT}, p}\left(\epsilon_{0}\right)$ for the AIP and PIP cases can be obtained as $\log \left(1+\gamma_{a}\right)$ and $\log \left(1+\gamma_{p}\right)$, respectively.

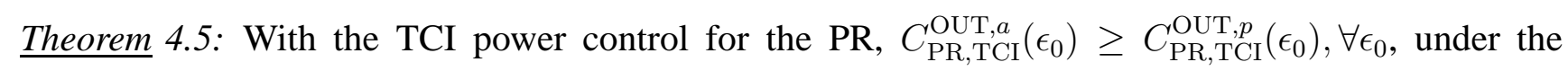
same average and peak power threshold $\Gamma$.

Proof: Similarly like the discussions for the PR outage capacity with the CP policy, we compare $C_{\mathrm{PR}, \mathrm{TCI}}^{\mathrm{OUT}, a}\left(\epsilon_{0}\right)$ and $C_{\mathrm{PR}, \mathrm{TCI}}^{\mathrm{OUT}, p}\left(\epsilon_{0}\right)$ via the following equivalent problem: Given $\gamma_{a}=\gamma_{p}=\gamma_{0}$, we compare the minimum outage probabilities in the AIP and PIP cases, denoted as $\epsilon_{a}$ and $\epsilon_{p}$, respectively. If $\epsilon_{a} \leq \epsilon_{p}, \forall \gamma_{0}$, we then conclude that $C_{\mathrm{PR}, \mathrm{TCI}}^{\mathrm{OUT}, a}\left(\epsilon_{0}\right) \geq C_{\mathrm{PR}, \mathrm{TCI}}^{\mathrm{OUT}, p}\left(\epsilon_{0}\right), \forall \epsilon_{0}$.

Next, we show that $\epsilon_{a} \leq \epsilon_{p}, \forall \gamma_{0}$. Similarly like the proof of Theorem 4.2, the Lagrange duality is applied here. For given $Q$ and $\gamma_{0}, \epsilon_{a}$ and $\epsilon_{p}$ can be rewritten as the optimal values of the following 
max-min optimization problems:

$$
\epsilon_{a}=\max _{\mu: \mu \geq 0} \min _{\left\{q_{i}\right\}: q_{i} \geq 0, \forall i} \operatorname{Pr}\left\{\frac{f_{i} q_{i}}{1+I_{i}}<\gamma_{0}\right\}+\mu\left(\mathbb{E}\left[q_{i}\right]-Q\right)
$$

and

$$
\epsilon_{p}=\max _{\mu: \mu \geq 0} \min _{\left\{q_{i}\right\}: q_{i} \geq 0, \forall i} \operatorname{Pr}\left\{\frac{f_{i} q_{i}}{1+\Gamma}<\gamma_{0}\right\}+\mu\left(\mathbb{E}\left[q_{i}\right]-Q\right)
$$

respectively. Note that $\mu_{a}=\theta_{a} / \gamma_{0}$ and $\left\{q_{i}^{\mathrm{TCI}, a}\right\}$ are the optimal solutions to the "max" and "min" problems in (33), respectively, and $\mu_{p}=\theta_{p} / \gamma_{0}$ and $\left\{q_{i}^{\mathrm{TCP}, p}\right\}$ are the optimal solutions to the "max" and "min" problems in (34), respectively. Then, we have the following equalities/inequalities:

$$
\begin{aligned}
& \epsilon_{p}=\max _{\mu: \mu \geq 0} \mathbb{E}_{f}\left[\mathbf{1}\left(\frac{f_{i}}{1+\Gamma}<\gamma_{0} \mu\right)\right]+\mu \mathbb{E}_{f}\left[\frac{(1+\Gamma) \gamma_{0}}{f_{i}} \mathbf{1}\left(\frac{f_{i}}{1+\Gamma} \geq \gamma_{0} \mu\right)\right]-\mu Q \\
& \geq \mathbb{E}_{f}\left[\mathbf{1}\left(\frac{f_{i}}{1+\Gamma}<\gamma_{0} \mu_{a}\right)\right]+\mu_{a} \mathbb{E}_{f}\left[\frac{(1+\Gamma) \gamma_{0}}{f_{i}} \mathbf{1}\left(\frac{f_{i}}{1+\Gamma} \geq \gamma_{0} \mu_{a}\right)\right]-\mu_{a} Q \\
& =1+\mathbb{E}_{f}\left[\left(\frac{(1+\Gamma) \gamma_{0} \mu_{a}}{f_{i}}-1\right) \mathbf{1}\left(\frac{f_{i}}{1+\Gamma} \geq \gamma_{0} \mu_{a}\right)\right]-\mu_{a} Q \\
& =1+\mathbb{E}_{f}\left[\left(\frac{\left(1+\mathbb{E}\left[I_{i}\right]\right) \gamma_{0} \mu_{a}}{f_{i}}-1\right) \mathbf{1}\left(\frac{f_{i}}{1+\mathbb{E}\left[I_{i}\right]} \geq \gamma_{0} \mu_{a}\right)\right]-\mu_{a} Q \\
& \geq 1+\mathbb{E}_{f} \mathbb{E}_{I}\left[\left(\frac{\left(1+I_{i}\right) \gamma_{0} \mu_{a}}{f_{i}}-1\right) \mathbf{1}\left(\frac{f_{i}}{1+I_{i}} \geq \gamma_{0} \mu_{a}\right)\right]-\mu_{a} Q \\
& =\epsilon_{a}
\end{aligned}
$$

where (35) is obtained by substituting $\left\{q_{i}^{\mathrm{TCI}, p}\right\}$ in (32) with $\theta_{p}$ replaced by $\gamma_{0} \mu$ into (34); (36) is due to the fact that $\mu_{a}$ is not the maximizer $\mu_{p}$ for $(35) ;(38)$ is due to $\mathbb{E}\left[I_{i}\right]=\Gamma$; 39 is due to concavity of the function in $\mathbb{E}_{f}[\cdot]$ of $(38)$ with respect to $\mathbb{E}\left[I_{i}\right]$ for any given $f_{i}$ and Jensen's inequality; (40) is due to the fact that $\mu_{a}$ and $\left\{q_{i}^{\mathrm{TCI}, a}\right\}$ in (31) are the optimal solutions to the the max-min optimization problem in (33).

Theorem 4.5 suggests that for the TCI power control of the PR, the interference diversity gain due to the AIP constraint over the PIP exists regardless of the outage probability. Note that in Theorem 4.4 for the extreme case of zero outage probability, it has been shown that the delay-limited capacities are the same for both the AIP and PIP constraints.

\section{Simulation Results and Discussions}

So far, we have studied the effect of the AIP and PIP constraints on the ergodic/outage capacity of the CR link and the PR link separately. In this section, we will consider a realistic spectrum sharing scenario 
over the fading channels, and evaluate by simulation the jointly achievable ergodic/outage capacities for both the PR and CR links. In total, we will consider four cases of different combinations, which are CR ergodic capacity versus PR ergodic capacity, CR ergodic capacity versus PR outage capacity, CR outage capacity versus PR ergodic capacity, and CR outage capacity versus PR outage capacity, in Figs. 2,5, respectively. It is assumed that $\Gamma_{a}=\Gamma_{p}=1$, the same as the additive Gaussian noise power at PR-Rx and CR-Rx. It is also assumed that $\boldsymbol{h}, \boldsymbol{g}$, and $\boldsymbol{f}$ are obtained from the Rayleigh fading model, i.e., they are the squared norms of independent $\mathrm{CSCG}$ r.v.s $\sim \mathcal{C N}(0,1), \mathcal{C N}(0,10)$, and $\mathcal{C N}(0,1)$, respectively. Note that we have purposely set the average power for $\boldsymbol{g}$ to be $10 \mathrm{~dB}$ larger than that for $\boldsymbol{h}$ or $\boldsymbol{f}$ so as to pronounce the effect of the interference channel from CR-Tx to PR-Rx on the achievable capacities. The PR transmit power constraint is set to be $Q=10$. In the cases of outage capacities of the PR and/or $\mathrm{CR}$, the outage probability targets of $\epsilon_{0}$ for PR and CR are set to be 0.2 and 0.1 , respectively. In each figure, the PR and CR capacities in bits/complex dimension (dim.) are plotted versus the additional channel power gain attenuation of $\boldsymbol{g}$ in $\mathrm{dB}$. For example, for 0 - $\mathrm{dB}$ attenuation, $\mathbb{E}\left[g_{i}\right]=10$; for 10 - $\mathrm{dB}$ attenuation, $\mathbb{E}\left[g_{i}\right]=1$.

In Figs. 2 and 3, we compare the CR ergodic capacity under AIP or PIP constraint with the corresponding ergodic and outage capacities for the PR, respectively. Note that the CR ergodic capacities shown in these two figures are the same. With increasing channel attenuation of $\boldsymbol{g}$, it is observed that the CR ergodic capacity increases for both AIP and PIP cases. This is obvious since given the fixed peak or average interference-power threshold at PR-Rx, decreasing of the average power for $\boldsymbol{g}$ results in increasing of the average transmit power of the CR. It is also observed that the AIP-based optimal power control performs better than the PIP-based one for the CR, since the former is more flexible for exploiting all the available CSI at CR-Tx. Interestingly, as the average power for $\boldsymbol{g}$ decreases, eventually the CR ergodic capacities in the AIP and PIP cases converge to the same value. This can be explained as follows. From (5) and (7), it follows that in the AIP case, the interference power at PR-Rx, $I_{i}$, is randomized over $i$ (but with $\mathbb{E}\left[I_{i}\right]=\Gamma$ ), while in the PIP case, $I_{i}$ is constantly equal to $\Gamma$ for each $i$. Note that the above fact leads to the interference diversity gain of the AIP over the PIP for the PR transmission. However, with $g_{i} \rightarrow 0$, it can be shown in the AIP case that $1 / \nu \rightarrow \Gamma$ and $I_{i} \rightarrow \Gamma$, which 
implies that $p_{i}^{\mathrm{ER}, a}=p_{i}^{\mathrm{ER}, p}=\Gamma / g_{i}, \forall i$, and thus the same CR ergodic capacity is resultant for both the AIP and PIP cases.

On the other hand, it is observed that the ergodic and outage capacities for the PR under the AIP from CR-Tx are larger than the corresponding ones under the PIP for various PR power-control policies, which are in accord with the analytical results obtained in Section IV. Note that the PR ergodic/outage capacities in the PIP case are fixed regardless of the channel power for $\boldsymbol{g}$, since $I_{i}$ is fixed as $\Gamma$ at PR-Rx for each $i$. However, the PR ergodic/outage capacities in the AIP case are observed to decrease with increasing of the channel attenuation of $\boldsymbol{g}$. This is due to the fact that, as explained earlier, $I_{i} \rightarrow \Gamma$ as $g_{i} \rightarrow 0$. Since the capacity gain of the AIP over the PIP is due to the randomness of $I_{i}$ over $i$, this interference diversity gain diminishes as $I_{i} \rightarrow \Gamma, \forall i$.

In Figs. 4 and 5, we compare the CR outage capacity under AIP or PIP constraint with the corresponding ergodic and outage capacities for the PR, respectively. Note that the CR outage capacities shown in these two figures are identical. With increasing channel attenuation of $\boldsymbol{g}$, it is observed that, as expected, the CR outage capacity increases for both AIP and PIP cases. It is also observed that the AIP-based optimal power control results in substantial outage capacity gains than the PIP-based one for the CR. It can be shown that as the average power for $\boldsymbol{g}$ decreases, eventually the CR outage capacity gaps between the AIP and the PIP cases converge to $\log \left(\zeta_{a} / \zeta_{p}\right)$ for a given $\epsilon_{0}$. The proof is given as follows. Suppose that $g_{i}^{\prime}=\kappa g_{i}, \forall i$, where $\kappa$ is a positive constant; we thus have $\mathbb{E}\left[g_{i}^{\prime}\right]=\kappa \mathbb{E}\left[g_{i}\right]$. For a given $\epsilon_{0}$, it then follows that the new value of threshold in (6) becomes $\lambda^{\prime}=\lambda / \kappa$. From (6) and under the same value of $\Gamma_{a}$, we have $\zeta_{a}^{\prime}=\zeta_{a} / \kappa$. Thus, the outage capacity corresponding to $\boldsymbol{g}^{\prime}$ in the AIP case is expressed as $\log \left(1+\zeta_{a} / \kappa\right)$. Similarly, we can show that in the PIP case, the new value of $\zeta_{p}$ corresponding to $\boldsymbol{g}^{\prime}$ is $\zeta_{p}^{\prime}=\zeta_{p} / \kappa$ and thus the corresponding outage capacity becomes $\log \left(1+\zeta_{p} / \kappa\right)$. Thus, the outage capacity gap between the AIP and PIP cases is equal to $\log \left(\frac{1+\zeta_{a} / \kappa}{1+\zeta_{p} / \kappa}\right)$. As $\kappa \rightarrow 0$, we conclude that the above capacity gap converges to $\log \left(\zeta_{a} / \zeta_{p}\right)$. Note that in this simulation with $\epsilon_{0}=0.1$ for the $\mathrm{CR}, \log \left(\zeta_{a} / \zeta_{p}\right)=2.6791$ bits/complex dimension.

Furthermore, it is observed that the ergodic and outage capacities for the PR under the AIP from CR-Tx are also larger than the corresponding ones under the PIP for various PR power-control policies, 
as have been analytically shown in Section IV. Note that not only the PR ergodic/outage capacities in the PIP case are fixed for all the average powers for $\boldsymbol{g}$ due to that $I_{i}$ is fixed as $\Gamma$ for each $i$, but also are these capacities in the AIP case. The latter observation can be explained by noting from the earlier proof that for any channel power gains $g_{i}^{\prime}, g_{i}^{\prime}=\kappa g_{i}, \forall i$, the resultant interference power at PR-Rx, $I_{i}^{\prime}$, can be shown to have the same distribution as $I_{i}$; as a result, the PR capacities are constant regardless of $\kappa$.

\section{CONCLUding REMARKS}

This paper studies the information-theoretic limits for wireless spectrum sharing in the PR-CR network where the CR applies the interference-power/interference-temperature constraint at the PR receiver as a practical means to protect the PR transmission. On the contrary to the traditional viewpoint that the peakinterference-power (PIP) constraint protects better the PR transmission than the average-interferencepower (AIP) constraint given their same power-threshold value, this paper shows that the AIP constraint can be in many cases more advantageous over the PIP for minimizing the resultant capacity losses of the PR fading channel. This is mainly owing to an interesting interference diversity phenomenon discovered in this paper. This paper thus provides an important design rule for the CR networks in practice, i.e., the AIP constraint should be used for the purposes of both protecting the PR transmission as well as maximizing the $\mathrm{CR}$ throughput.

This paper assumes that the perfect CSI on the interference channel from the CR transmitter to the PR receiver is available at the CR transmitter for each fading state. In practice, it is usually more valid to assume availability of only the statistical channel knowledge. The definition of the AIP constraint in this paper can be extendible to such cases. Furthermore, this paper considers the fading PR and CR channels, but more generally, the results obtained also apply to other channel models consisting of parallel Gaussian channels over which the average and peak power constraints are applicable, e.g., the time-dispersive broadband channel that is decomposable into parallel narrow-band channels by the well-known orthogonal-frequency-division-multiplexing (OFDM) modulation/demodulation.

\section{REFERENCES}

[1] Joseph Mitola, "Cognitive radio: an integrated agent architecture for software defined radio," PhD Dissertation, KTH, Stockholm, Sweden, Dec. 2000. 
[2] N. Devroye, P. Mitran, and V. Tarokh, "Achievable rates in cognitive radio channels," IEEE Trans. Inf. Theory, vol. 52, no. 5, pp. 1813-1827, May 2006.

[3] A. Jovičić and P. Viswanath, "Cognitive radio: An information-theoretic perspective," Proc. IEEE Int. Symp. Inf. Theory (ISIT), Jul. 2006.

[4] S. Haykin, "Cognitive radio: brain-empowered wireless communications," IEEE J. Sel. Areas Commun., vol. 23, no. 2, pp. 201-220, Feb. 2005.

[5] M. Gastpar, "On capacity under receive and spatial spectrum-sharing constraints," IEEE Trans. Inf. Theory, vol. 53, no. 2, pp. 471-487, Feb. 2007.

[6] A. Ghasemi and E. S. Sousa, "Fundamental limits of spectrum-sharing in fading environments," IEEE Trans. Wireless Commun., vol. 6, no. 2, pp. 649-658, Feb. 2007.

[7] L. Musavian and S. Aissa, "Ergodic and outage capacities of spectrum-sharing systems in fading channels," in Proc. IEEE Global Commun. Conf. (Globecom), Nov. 2007.

[8] X. Kang, Y. C. Liang, N. Arumugam, H. Garg, and R. Zhang , Optimal power allocation for fading channels in cognitive radio networks: ergodic capacity and outage capacity," to appear in IEEE Trans. Wireless Commun., also available at arXiv:0808.3689

[9] R. Zhang, "Optimal power control over fading cognitive radio channels by exploiting primary user CSI", to appear in IEEE Global Commun. Conf. (Globecom), also available at arXiv:0804.1617

[10] Y. Chen G. Yu, Z. Zhang, H. H. Chen, and P. Qiu, "On cognitive radio networks with opportunistic power control strategies in fading channels," IEEE. Trans. Wireless Commun., vol. 7, no. 7, pp. 2752-2761, Jul. 2008.

[11] R. Zhang and Y. C. Liang, "Exploiting multi-antennas for opportunistic spectrum sharing in cognitive radio networks," IEEE J. S. Topics Sig. Proc., vol. 2, no. 1, pp. 88-102, Feb. 2008.

[12] L. Zhang, Y. C. Liang, and Y. Xin, "Joint beamforming and power control for multiple access channels in cognitive radio networks," IEEE J. Sel. Areas Commun., vol.26, no.1, pp.38-51, Jan. 2008.

[13] R. Zhang, S. Cui, and Y. C. Liang, "On ergodic sum capacity of fading cognitive multiple-access and broadcast channels," submitted to IEEE Trans. Inf. Theory, also available at arXiv:0806.4468

[14] T. Cover and J. Thomas, Elements of Information Theory, New York: Wiley, 1991.

[15] A. Goldsmith and P. P. Varaiya, "Capacity of fading channels with channel side information," IEEE Trans. Inf. Theory, vol. 43, no. 6, pp. 1986-1992, Nov. 1997.

[16] L. H. Ozarow, S. Shamai, and A. D. Wyner, "Information theoretic considerations for cellular mobile radio," IEEE Trans. Veh. Technol., vol. 43 no. 2, pp. 359-378, 1994.

[17] G. Caire, G. Taricco, and E. Biglieri, "Optimal power control over fading channels," IEEE Trans. Inf. Theory, vol. 45, no. 5, pp. 1468-1489, Jul. 1999.

[18] S. Hanly and D. Tse,"Multi-access fading channels-Part II: Delay-limited capacities," IEEE Trans. Inf. Theory, vol. 44, no.7, pp. 2816-2831, Nov. 1998.

[19] S. Boyd and L. Vandenberghe, Convex optimization, Cambridge University Press, 2004. 


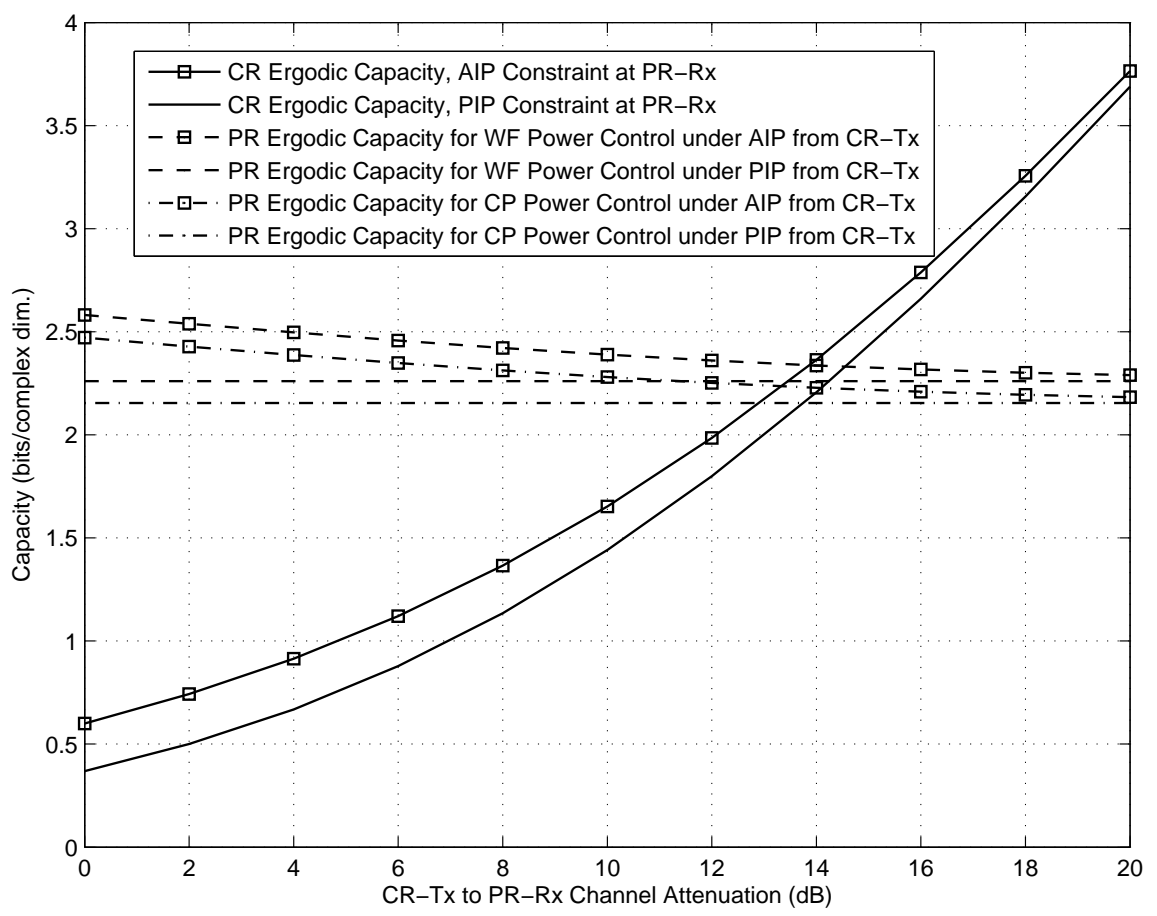

Fig. 2. Jointly achievable CR ergodic capacity and PR ergodic capacity.

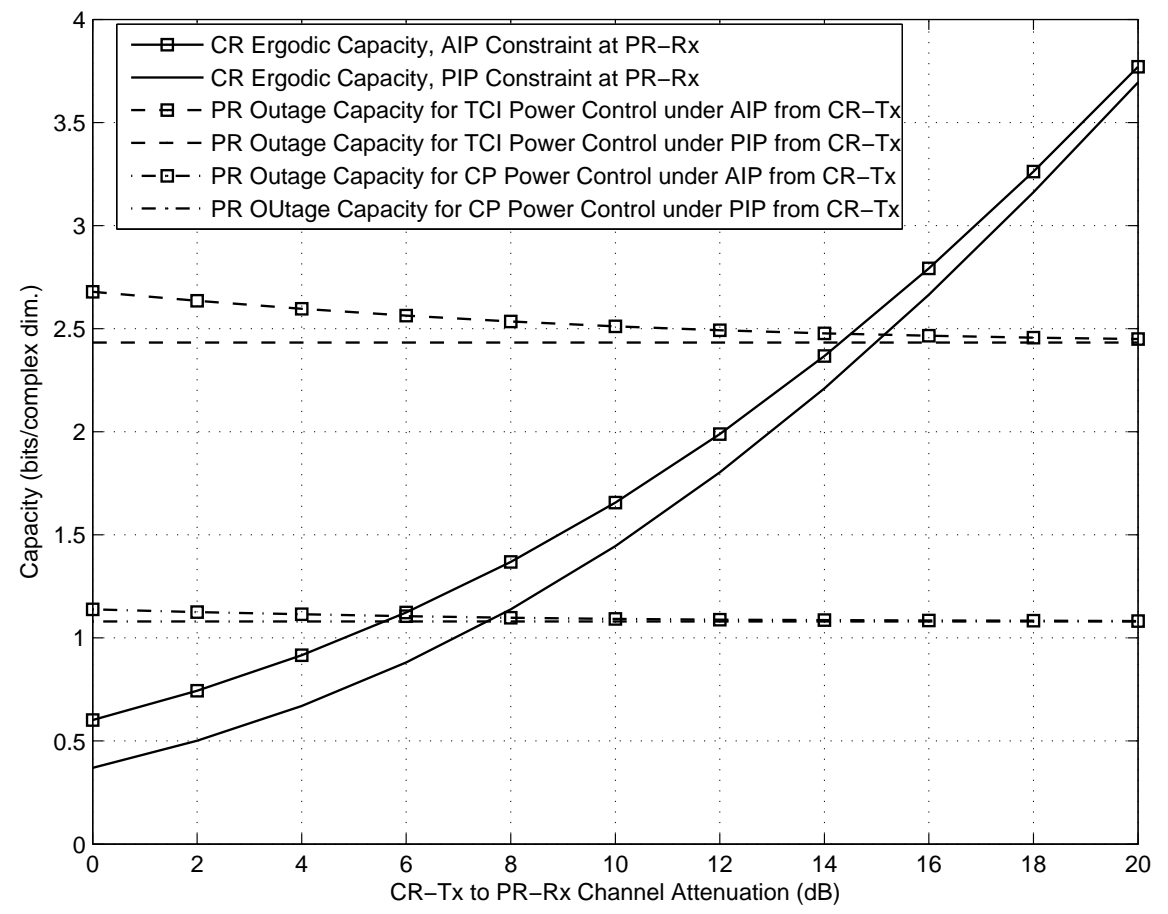

Fig. 3. Jointly achievable CR ergodic capacity and PR outage capacity. 


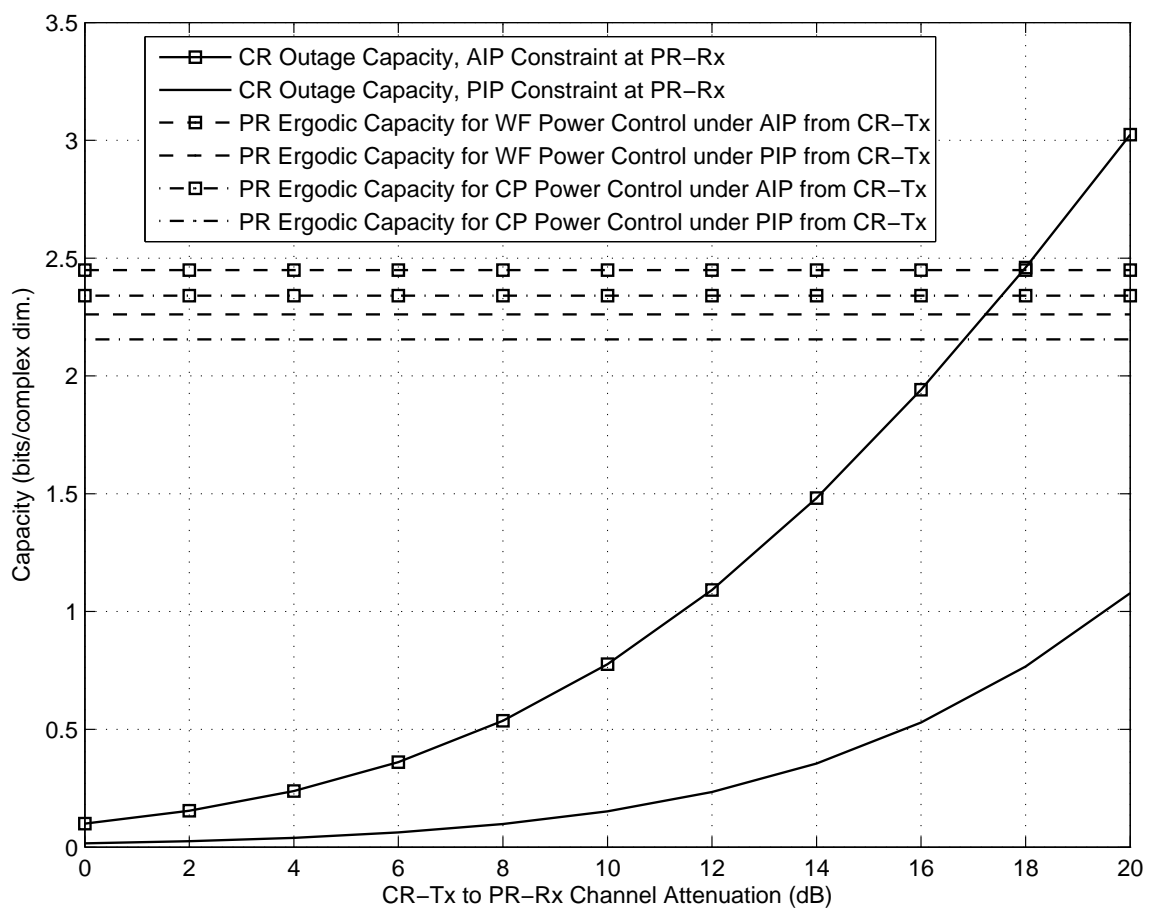

Fig. 4. Jointly achievable CR outage capacity and PR ergodic capacity.

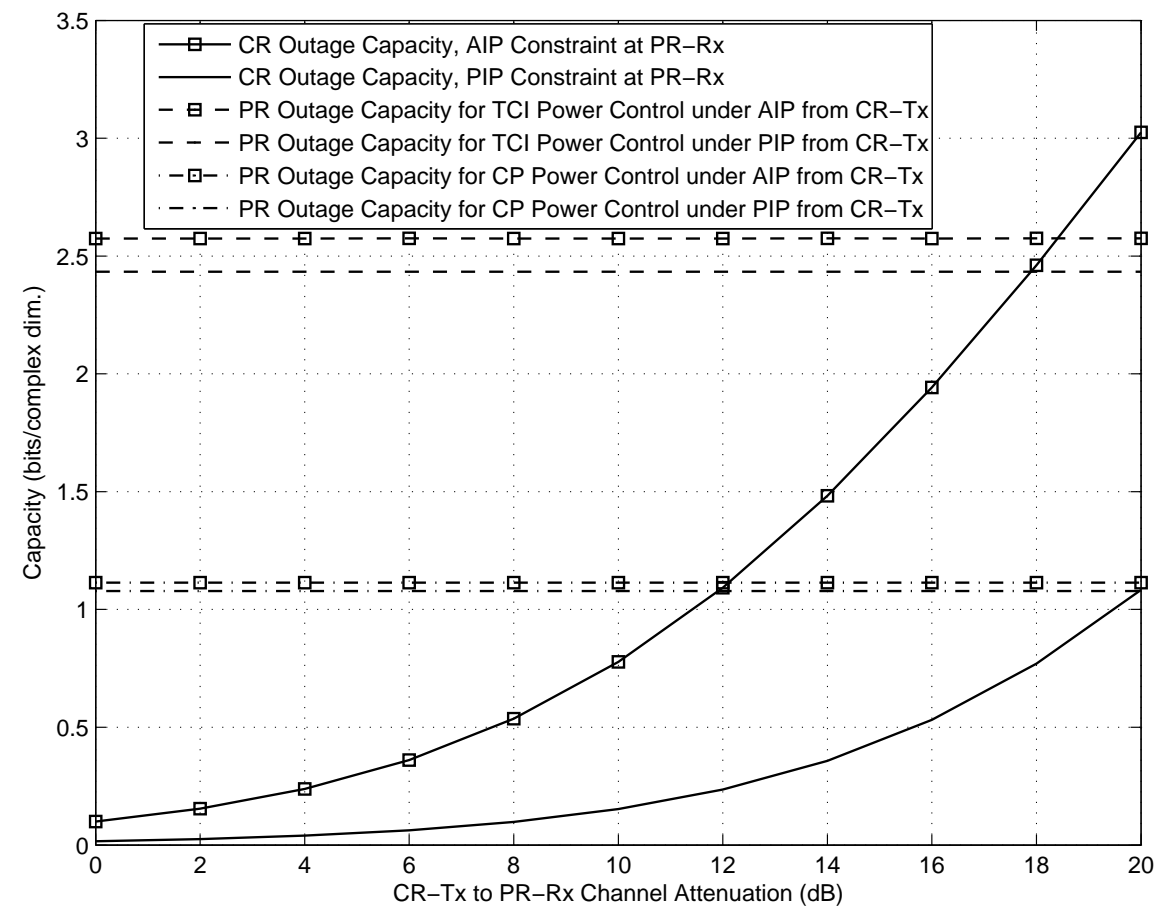

Fig. 5. Jointly achievable CR outage capacity and PR outage capacity. 